International Journal of Computational Geometry \& Applications

(c) World Scientific Publishing Company

\title{
$\pi / 2$-ANGLE YAO GRAPHS ARE SPANNERS
}

\author{
PROSENJIT BOSE \\ School of Computer Science, Carleton University \\ Ottawa, Canada \\ jit@scs.carleton.ca \\ MIRELA DAMIAN \\ Department of Computer Science, Villanova University \\ Villanova, USA \\ mirela.damian@villanova.edu \\ KARIM DOUÏEB \\ School of Computer Science, Carleton University \\ Ottawa, Canada \\ kdouieb@ulb.ac.be \\ JOSEPH O'ROURKE \\ Department of Computer Science, Smith College \\ Northampton, USA \\ orourke@cs.smith.edu \\ BEN SEAMONE \\ School of Mathematics and Statistics, Carleton University \\ Ottawa, Canada \\ bseamone@connect.carleton.ca \\ MICHIEL SMID \\ School of Computer Science, Carleton University \\ Ottawa, Canada \\ michiel@scs.carleton.ca \\ STEFANIE WUHRER \\ Institute for Information Technology, National Research Council \\ Ottawa, Canada \\ stefanie.wuhrer@nrc-cnrc.gc.ca
}

We show that the Yao graph $Y_{4}$ in the $L_{2}$ metric is a spanner with stretch factor $8 \sqrt{2}(26+$ $23 \sqrt{2}$ ). Enroute to this, we also show that the Yao graph $Y_{4}^{\infty}$ in the $L_{\infty}$ metric is a plane spanner with stretch factor 8 .

Keywords: Yao graph; Y4; spanner. 


\section{Introduction}

Let $V$ be a finite set of points in the plane and let $G=(V, E)$ be the complete Euclidean graph on $V$. We will refer to the points in $V$ as nodes, to distinguish them from other points in the plane. The $Y a o$ graph ${ }^{8}$ with an integer parameter $k>0$, denoted $Y_{k}$, is defined as follows. Any $k$ equally-separated rays starting at the origin define $k$ cones. Pick a set of arbitrary, but fixed cones. Translate the cones to each node $u \in V$. In each cone with apex $u$, pick a shortest edge $u v$, if there is one, and add to $Y_{k}$ the directed edge $\overrightarrow{u v}$. Ties are broken arbitrarily. Note that the Yao graph differs from the $\Theta$-graph in how the shortest edge is chosen. While the Yao graph chooses the shortest edge in terms of the Euclidean distance, the $\Theta$-graph chooses the edge whose projection on the bisector of the cone is shortest. Most of the time we ignore the direction of an edge $u v$; we refer to the directed version $\overrightarrow{u v}$ of $u v$ only when its origin $(u)$ is important and unclear from the context. We will distinguish between $Y_{k}$, the Yao graph in the Euclidean $L_{2}$ metric, and $Y_{k}^{\infty}$, the Yao graph in the $L_{\infty}$ metric. Unlike $Y_{k}$ however, in constructing $Y_{k}^{\infty}$ ties are broken by always selecting the most counterclockwise edge; the reason for this choice will become clear in Section 2.

The length of a path is the sum of the lengths of its constituent edges. For a given subgraph $H \subseteq G$ and a fixed $t \geq 1, H$ is called a $t$-spanner for $G$ if, for any two nodes $u, v \in V$, the shortest path in $H$ from $u$ to $v$ is no longer than $t$ times the length $|u v|$ of $u v$. The value $t$ is called the dilation or the stretch factor of $H$. If $t$ is constant, then $H$ is called a length spanner, or simply a spanner.

The class of graphs $Y_{k}$ has been much studied. Bose et al. ${ }^{2}$ showed that, for $k \geq$ $9, Y_{k}$ is a spanner with stretch factor $\frac{1}{\cos \frac{2 \pi}{k}-\sin \frac{2 \pi}{k}}$. In Ref. ${ }^{1}$ we improved the stretch factor and showed that, in fact, $Y_{k}$ is a spanner for any $k \geq 7$. Recently, Damian and Raudonis ${ }^{4}$ showed that $Y_{6}$ is a 17.7-spanner. Molla ${ }^{6}$ showed that $Y_{2}$ and $Y_{3}$ are not spanners, and that $Y_{4}$ is a spanner with stretch factor $4(2+\sqrt{2})$, for the special case when the nodes in $V$ are in convex position (see also Ref. ${ }^{3}$ ). The authors conjectured that $Y_{4}$ is a spanner for arbitrary point sets. In this paper, we settle their conjecture and prove that $Y_{4}$ is a spanner with stretch factor $8 \sqrt{2}(26+23 \sqrt{2})$.

The paper is organized as follows. In Section 2, we prove that the graph $Y_{4}^{\infty}$ is a spanner with stretch factor 8 . In Section 3 we establish several properties for the graph $Y_{4}$. Finally, in Section 4, we use the properties of Section 3 to prove that, for every edge $a b$ in $Y_{4}^{\infty}$, there exists a path between $a$ and $b$ in $Y_{4}$ not much longer than the Euclidean distance between $a$ and $b$. By combining this with the result of Section 2, we conclude that $Y_{4}$ is a spanner.

\section{2. $Y_{4}^{\infty}$ in the $L_{\infty}$ Metric}

In this section we focus on $Y_{4}^{\infty}$, which has a nicer structure compared to $Y_{4}$. First we prove that $Y_{4}^{\infty}$ is a plane graph. Then we use this property to show that $Y_{4}^{\infty}$ is an 8-spanner. To be more precise, we prove that for any two nodes $a$ and $b$, the graph $Y_{4}^{\infty}$ contains a path between $a$ and $b$ whose length (in the $L_{\infty}$-metric) is at 
most $8|a b|_{\infty}$.

We need a few definitions. We say that two edges $a b$ and $c d$ properly cross (or cross, for short) if they share a point other than an endpoint $(a, b, c$ or $d)$; we say that $a b$ and $c d$ intersect if they share a point (either an interior point or an endpoint).

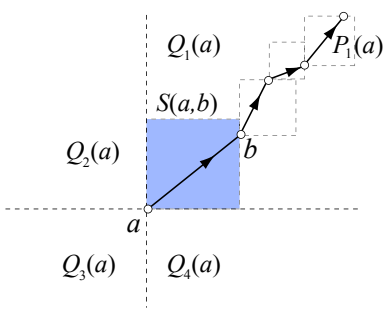

(a)

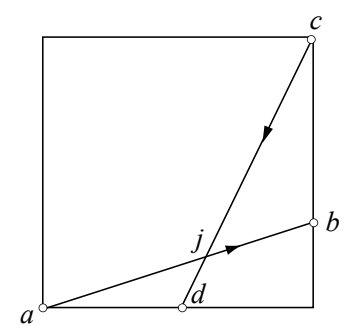

(b)

Fig. 1. (a) Definitions: $Q_{i}(a), P_{i}(a)$ and $S(a, b)$. (b) Lemma 1: $a b$ and $c d$ cannot cross.

Throughout the paper, we will use the following notation: for each node $a \in V$, $x(a)$ is the $x$-coordinate of $a$ and $y(a)$ is the $y$-coordinate of $a ; Q_{1}(a), Q_{2}(a), Q_{3}(a)$ and $Q_{4}(a)$ are the four quadrants at $a$, depicted in Fig. 1a; each quadrant is half-open and half-closed, including all points on the clockwise boundary axis (with respect to the quadrant bisector through $a$ ), and excluding all points on the counterclockwise boundary axis; $P_{i}(a)$ is the path that starts at $a$ and follows the directed Yao edges in quadrant $Q_{i} ; P_{i}(a, b)$ is the subpath of $P_{i}(a)$ that starts at node $a$ and ends at node $b$; $|a b|_{\infty}$ is the $L_{\infty}$ distance between $a$ and $b$, defined as $\max \{|x(a)-x(b)|,|y(a)-y(b)|\}$; $s p(a, b)$ is a shortest path in $Y_{4}^{\infty}$ between $a$ and $b ; S(a, b)$ is the open square with corner $a$ whose boundary contains $b$; and $\partial S(a, b)$ is the boundary of $S(a, b)$. These definitions are depicted in Fig. 1a.

Lemma 1. $Y_{4}^{\infty}$ is a plane graph.

Proof. The proof is by contradiction. Assume the opposite. Then there are two edges $\overrightarrow{a b}, \overrightarrow{c d} \in Y_{4}^{\infty}$ that cross each other. Since $\overrightarrow{a b} \in Y_{4}^{\infty}, S(a, b)$ must be empty of nodes in $V$, and similarly for $S(c, d)$. Let $j$ be the intersection point between $a b$ and $c d$. Then $j \in S(a, b) \cap S(c, d)$, meaning that $S(a, b)$ and $S(c, d)$ must overlap. However, neither square may contain $a, b, c$ or $d$. It follows that $S(a, b)$ and $S(c, d)$ coincide, meaning that $c$ and $d$ lie on $\partial S(a, b)$ (see Fig. 1b). Since $c d$ intersects $a b$, $c$ and $d$ must lie on opposite sides of $a b$. Thus either $a c$ or $a d$ lies counterclockwise from $a b$. Assume without loss of generality that $a c$ lies counterclockwise from $a b$; the other case is identical. Because $S(a, c)$ coincides with $S(a, b)$, we have that $|a c|_{\infty}=|a b|_{\infty}$. In this case however, $Y_{4}^{\infty}$ would break the tie between $a c$ and $a b$ by selecting the most counterclockwise edge, which is $\overrightarrow{a c}$. This contradicts that $\overrightarrow{a b} \in Y_{4}^{\infty}$. 
Theorem 1. $Y_{4}^{\infty}$ is an 8-spanner in the $L_{\infty}$ metric.

Proof. We show that, for any pair of points $a, b \in V,|s p(a, b)|_{\infty}<8|a b|_{\infty}$. The proof is by induction on the pairwise $L_{\infty}$-distance between the points in $V$. Assume without loss of generality that $b \in Q_{1}(a)$, and $|a b|_{\infty}=|x(b)-x(a)|$ (i.e., $b$ lies below the diagonal of $S(a, b)$ incident to $a$ ). Consider the case in which $a b$ is a closest (in the $L_{\infty}$ metric) pair of points in $V$. This is the base case for our induction. If $a b \in Y_{4}^{\infty}$, then $|s p(a, b)|_{\infty}=|a b|_{\infty}$. Otherwise, there must be $a c \in Y_{4}^{\infty}$, with $|a c|_{\infty}=|a b|_{\infty}$. Recall that $Y_{4}^{\infty}$ breaks ties by always selecting the most counterclockwise edge, so $a c$ must be counterclockwise of $a b$. Also recall that $Q_{1}(a)$ does not include the vertical coordinate axis through $a$, therefore $c$ lies strictly to the right of $a$. It follows that $|b c|_{\infty}<|a b|_{\infty}$ (see Fig. 2a), a contradiction.

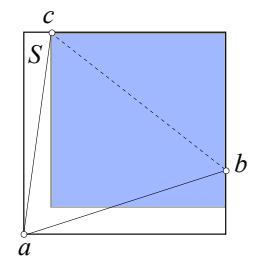

(a)

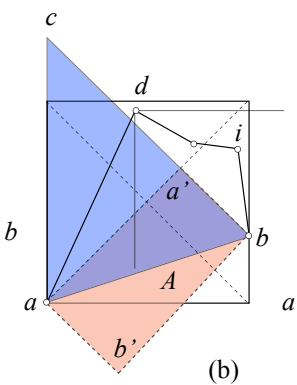

(b)

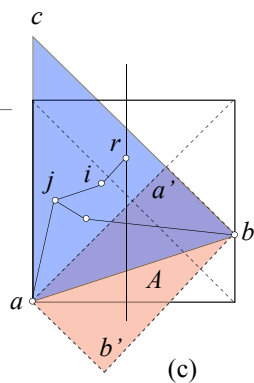

(c)

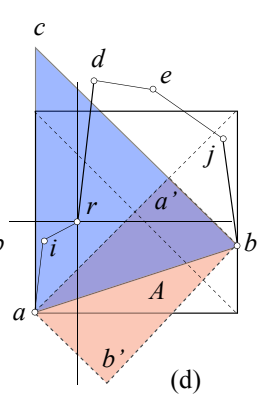

(d)

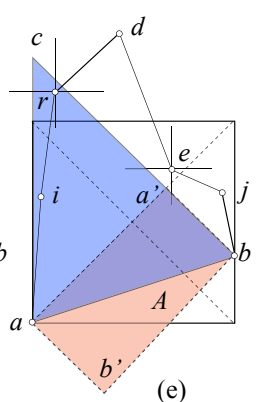

(e)

Fig. 2. (a) Base case. (b) $\triangle a b c$ empty (c) $\triangle a b c$ non-empty, $P_{a r} \cap P_{2}(b)=\{j\}$ (d) $\triangle a b c$ non-empty, $P_{a r} \cap P_{2}(b)=\emptyset$, e above $r$ (e) $\triangle a b c$ non-empty, $P_{a r} \cap P_{2}(b)=\emptyset$, e below $r$.

Assume now that the inductive hypothesis holds for all pairs of points closer (in the $L_{\infty}$ metric) than $|a b|_{\infty}$. If $a b \in Y_{4}^{\infty}$, then $|s p(a, b)|_{\infty}=|a b|_{\infty}$ and the proof is finished. If $a b \notin Y_{4}^{\infty}$, then the square $S(a, b)$ must be nonempty.

Let $A$ be the rectangle $a b^{\prime} b a^{\prime}$ as in Fig. 2b, where $b a^{\prime}$ and $b b^{\prime}$ are parallel to the diagonals of $S(a, b)$. If $A$ is nonempty, then we can use induction to prove that $|s p(a, b)|_{\infty} \leq 8|a b|_{\infty}$ as follows. Pick $c \in A$ arbitrary. Then $|a c|_{\infty}+|c b|_{\infty}=$ $|x(c)-x(a)|+|x(b)-x(c)|=|a b|_{\infty}$, and by the inductive hypothesis $\operatorname{sp}(a, c) \oplus \operatorname{sp}(c, b)$ is a path in $Y_{4}^{\infty}$ no longer than $8|a c|_{\infty}+8|c b|_{\infty}=8|a b|_{\infty}$; here $\oplus$ represents the concatenation operator. Assume now that $A$ is empty. Let $c$ be at the intersection between the line supporting $b a^{\prime}$ and the vertical line through $a$ (see Fig. 2b). We discuss two cases, depending on whether $\triangle a b c$ is empty of points or not.

Case 1: $\triangle a b c$ is empty of points. Let $a d \in P_{1}(a)$. We show that $P_{4}(d)$ cannot contain an edge crossing $a b$. Assume the opposite, and let $s t \in P_{4}(d)$ cross $a b$. Note that $s t \in P_{4}(d)$ also implies $s t \in P_{4}(s)$, which along with the fact that $s t$ crosses $a b$, implies that $s$ is either vertically aligned, or to the left of $b$.. Since $\triangle a b c$ is empty, $s$ must lie above $b c$ and $t$ below $a b$. It follows that $b$ and $t$ are in the same quadrant 
$Q_{4}(s)$ (recall that this quadrant includes the downward ray from $s$ ). Furthermore, $|s t|_{\infty} \geq|y(s)-y(t)|>|y(s)-y(b)|=|s b|_{\infty}$, contradicting the fact that $s t \in Y_{4}^{\infty}$.

We have established that $P_{4}(d)$ does not cross $a b$, which implies that $P_{4}(d)$ must exit $S(d, b)$ through its right edge. Also note that $P_{2}(b)$ cannot cross $a c$, because $\triangle a b c$ is empty of points, and any point left of $a c$ is $L_{\infty}$-farther from $b$ than $d$. It follows that $P_{2}(b)$ exits $S(b, d)$ through its top edge. This together with the fact that $P_{4}(d)$ exits $S(d, b)$ through its right edge, implies that $P_{4}(d)$ and $P_{2}(b)$ must meet in a point $i \in P_{4}(d) \cap P_{2}(b)$ (see Fig. 2b). Now note that $\left|P_{4}(d, i) \oplus P_{2}(b, i)\right|_{\infty} \leq$ $|x(d)-x(b)|+|y(d)-y(b)|<2|a b|_{\infty}$. Thus we have that $|\operatorname{sp}(a, b)|_{\infty} \leq \mid a d \oplus P_{4}(d, i) \oplus$ $\left.P_{2}(b, i)\right|_{\infty}<|a b|_{\infty}+2|a b|_{\infty}=3|a b|_{\infty}$.

Case 2: $\triangle a b c$ is nonempty. In this case, we seek a short path from $a$ to $b$ that does not cross to the underside of $a b$, to avoid oscillating paths that cross $a b$ arbitrarily many times. Let $r$ be the rightmost point that lies inside $\triangle a b c$. Arguments similar to the ones used in Case 1 show that $P_{3}(r)$ cannot cross $a b$ and therefore it must meet $P_{1}(a)$ in a point $i$. Then $P_{a r}=P_{1}(a, i) \oplus P_{3}(r, i)$ is a path in $Y_{4}^{\infty}$ of length

$$
\left|P_{a r}\right|_{\infty}<|x(a)-x(r)|+|y(a)-y(r)|<|a b|_{\infty}+2|a b|_{\infty}=3|a b|_{\infty} .
$$

The term $2|a b|_{\infty}$ in the inequality above results from the fact that $|y(a)-y(r)| \leq$ $|y(a)-y(c)| \leq 2|a b|_{\infty}$. Consider first the simpler situation in which $P_{2}(b)$ meets $P_{a r}$ in a point $j \in P_{2}(b) \cap P_{a r}$ (see Fig. 2c). Let $P_{a r}(a, j)$ be the subpath of $P_{a r}$ extending between $a$ and $j$. Then $P_{a r}(a, j) \oplus P_{2}(b, j)$ is a path in $Y_{4}^{\infty}$ from $a$ to $b$, therefore $|s p(a, b)|_{\infty} \leq\left|P_{a r}(a, j) \oplus P_{2}(b, j)\right|_{\infty}<2|y(j)-y(a)|+|a b|_{\infty} \leq 5|a b|_{\infty}$.

Consider now the case when $P_{2}(b)$ does not intersect $P_{a r}$. We argue that, in this case, $Q_{1}(r)$ may not be empty. Assume the opposite. Then no edge $s t \in P_{2}(b)$ may cross $Q_{1}(r)$. This is because, for any such edge, $|s r|_{\infty}<|s t|_{\infty}$, contradicting st $\epsilon$ $Y_{4}^{\infty}$. This implies that $P_{2}(b)$ intersects $P_{a r}$, again a contradiction to our assumption. This establishes that $Q_{1}(r)$ is nonempty. Let $r d \in P_{1}(r)$. The fact that $P_{2}(b)$ does not intersect $P_{a r}$ implies that $d$ lies to the left of $b$. The fact that $r$ is the rightmost point in $\triangle a b c$ implies that $d$ lies outside $\triangle a b c$ (see Fig. 2d). It also implies that $P_{4}(d)$ shares no points with $\triangle a b c$. This along with arguments similar to the ones used in case 1 show that $P_{4}(d)$ and $P_{2}(b)$ meet in a point $j \in P_{4}(d) \cap P_{2}(b)$. Thus we have found a path

$$
P_{a b}=P_{1}(a, i) \oplus P_{3}(r, i) \oplus r d \oplus P_{4}(d, j) \oplus P_{2}(b, j) .
$$

extending from $a$ to $b$ in $Y_{4}^{\infty}$. If $|r d|_{\infty}=|x(d)-x(r)|$, then $|r d|_{\infty}<|x(b)-x(a)|=$ $|a b|_{\infty}$, and the path $P_{a b}$ has length

$$
\left|P_{a b}\right|_{\infty} \leq 2|y(d)-y(a)|+|a b|_{\infty}<7|a b|_{\infty} .
$$

In the above, we used the fact that $|y(d)-y(a)|=|y(d)-y(r)|+|y(r)-y(a)|<$ $|a b|_{\infty}+2|a b|_{\infty}$. Suppose now that

$$
|r d|_{\infty}=|y(d)-y(r)|
$$


In this case, it is unclear whether the path $P_{a b}$ defined by (2) is short, since $r d$ can be arbitrarily long compared to $a b$. Let $e$ be the clockwise neighbor of $d$ along the path $P_{a b}$ ( $e$ and $b$ may coincide). Then $e$ lies below $d$, and either $d e \in P_{4}(d)$, or $e d \in P_{2}(e)$ (or both). If $e$ lies above $r$, or at the same level as $r$ (i.e., $e \in Q_{1}(r)$, as in Fig. 2d), then

$$
|y(e)-y(r)|<|y(d)-y(r)| \text {. }
$$

Since $r d \in P_{1}(r)$ and $e$ is in the same quadrant of $r$ as $d$, we have $|r d|_{\infty} \leq|r e|_{\infty}$. This along with inequalities (4) and (5) implies $|r e|_{\infty}>|y(e)-y(r)|$, which in turn implies $|r e|_{\infty}=|x(e)-x(r)| \leq|a b|_{\infty}$, and so $|r d|_{\infty} \leq|a b|_{\infty}$. Then inequality (3) applies here as well, showing that $\left|P_{a b}\right|_{\infty}<7|a b|_{\infty}$.

If $e$ lies below $r$ (as in Fig. 2e), then

$$
|e d|_{\infty} \geq|y(d)-y(e)| \geq|y(d)-y(r)|=|r d|_{\infty} .
$$

Assume first that $e d \in P_{2}(e)$, or $|e d|_{\infty}=|x(e)-x(d)|$. In either case, $|e d|_{\infty} \leq$ $|e r|_{\infty}<2|a b|_{\infty}$. This along with inequality (6) shows that $|r d|_{\infty}<2|a b|_{\infty}$. Substituting this upper bound in (2), we get $\left|P_{a b}\right|_{\infty} \leq 2|y(d)-y(a)|+2|a b|_{\infty}<8|a b|_{\infty}$. Assume now that $e d \notin P_{2}(e)$, and $|e d|_{\infty}=|y(e)-y(d)|$. Then $e e^{\prime} \in P_{2}(e)$ cannot go above $d$ (otherwise $|e d|_{\infty}<\left|e e^{\prime}\right|_{\infty}$, contradicting $e e^{\prime} \in P_{2}(e)$ ). This along with the fact $d e \in P_{4}(d)$ implies that $P_{2}(e)$ intersects $P_{a r}$ in a point $k$. Redefine $P_{a b}=P_{a r}(a, k) \oplus P_{2}(e, k) \oplus P_{4}(e, j) \oplus P_{2}(b, j)$. Then $P_{a b}$ is a path in $Y_{4}^{\infty}$ from $a$ to $b$ of length $\left|P_{a b}\right| \leq 2|y(r)-y(a)|+|a b|_{\infty} \leq 5|a b|_{\infty}$.

This theorem will be employed in Section 4.

\section{3. $Y_{4}$ in the $L_{2}$ Metric}

In this section we establish basic properties of $Y_{4}$. The ultimate goal of this section is to show that, if two edges in $Y_{4}$ cross, there is a short path between their endpoints (Lemma 8). We begin with a few definitions.

Let $Q(a, b)$ denote the infinite quadrant with origin at $a$ that contains $b$. For a pair of nodes $a, b \in V$, define recursively a directed path $\mathcal{P}(a \rightarrow b)$ from $a$ to $b$ in $Y_{4}$ as follows. If $a=b$, then $\mathcal{P}(a \rightarrow b)=$ null. If $a \neq b$, there must exist $\overrightarrow{a c} \in Y_{4}$ that lies in $Q(a, b)$. In this case, define

$$
\mathcal{P}(a \rightarrow b)=\overrightarrow{a c} \oplus \mathcal{P}(c \rightarrow b) .
$$

Recall that $\oplus$ represents the concatenation operator. This definition is illustrated in Fig. 3a. Fischer et al. ${ }^{5}$ show that $\mathcal{P}(a \rightarrow b)$ is well defined and lies entirely inside the square centered at $b$ whose boundary contains $a$.

For any path $P$ and any pair of nodes $a, b \in P$, let $P[a, b]$ be the subpath of $P$ from $a$ to $b$. Let $R(a, b)$ be the closed axis-aligned rectangle with diagonal $a b$ " (we permit $R(a, b)$ to be degenerate rectangle, when $a b$ is either horizontal or vertical).

For a fixed pair of nodes $a, b \in V$, define a path $\mathcal{P}_{R}(a \rightarrow b)$ as follows. Let $e \in V$ be the first node along $\mathcal{P}(a \rightarrow b)$ that is not strictly interior to $R(a, b)$. Then 


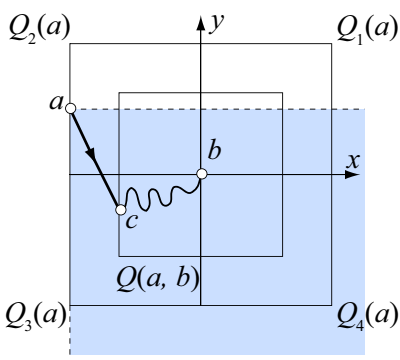

(a)

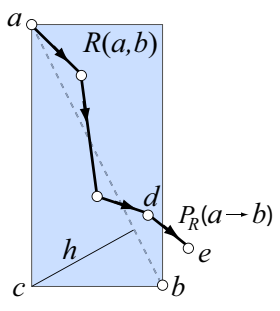

(b)

Fig. 3. Definitions. (a) $Q(a, b)$ and $\mathcal{P}(a \rightarrow b)$. (b) $\mathcal{P}_{R}(a \rightarrow b)$.

$\mathcal{P}_{R}(a \rightarrow b)$ is the subpath of $\mathcal{P}(a \rightarrow b)$ that extends between $a$ and $e$. In other words, $\mathcal{P}_{R}(a \rightarrow b)$ is the path that follows the $Y_{4}$ edges pointing towards $b$, truncated as soon as it reaches $b$ or leaves $R(a, b)$. Formally, $\mathcal{P}_{R}(a \rightarrow b)=\mathcal{P}(a \rightarrow b)[a, e]$. This definition is illustrated in Fig. 3b. Our proofs will make use of the following two propositions.

Proposition 1. The sum of the lengths of crossing diagonals of a non-degenerate (necessarily convex) quadrilateral abcd is strictly greater than the sum of the lengths of either pair of opposite sides:

$$
\begin{aligned}
& |a c|+|b d|>|a b|+|c d| \\
& |a c|+|b d|>|b c|+|d a| .
\end{aligned}
$$

This can be proved by partitioning the diagonals into two pieces each at their intersection point, and then applying the triangle inequality twice.

Proposition 2. For any triangle $\triangle a b c$, the following inequalities hold:

$$
|a c|^{2} \begin{cases}<|a b|^{2}+|b c|^{2}, & \text { if } \angle a b c<\pi / 2 \\ =|a b|^{2}+|b c|^{2}, & \text { if } \angle a b c=\pi / 2 \\ >|a b|^{2}+|b c|^{2}, & \text { if } \angle a b c>\pi / 2\end{cases}
$$

This proposition follows immediately from the Law of Cosines applied to triangle $\triangle a b c$.

Lemma 2. For each pair of nodes $a, b \in V$,

$$
\left|\mathcal{P}_{R}(a \rightarrow b)\right| \leq|a b| \sqrt{2} .
$$

Furthermore, each edge of $\mathcal{P}_{R}(a \rightarrow b)$ is no longer than $|a b|$.

Proof. Let $c$ be one of the two corners of $R(a, b)$, other than $a$ and $b$. Let $\overrightarrow{d e} \in$ $\mathcal{P}_{R}(a \rightarrow b)$ be the last edge on $\mathcal{P}_{R}(a \rightarrow b)$, which necessarily intersects $\partial R(a, b)$ 
(note that it is possible that $e=b$ ). Refer to Fig. 3b. Then $|d e| \leq|d b|$, otherwise $\overrightarrow{d e}$ could not be in $Y_{4}$. Since $d b$ lies in the rectangle with diagonal $a b$, we have that $|d b| \leq|a b|$, and similarly for each edge on $\mathcal{P}_{R}(a \rightarrow b)$. This establishes the latter claim of the lemma. For the first claim of the lemma, let $p=\mathcal{P}_{R}(a \rightarrow b)[a, d] \oplus d b$. Since $|d e| \leq|d b|$, we have that $\left|\mathcal{P}_{R}(a \rightarrow b)\right| \leq|p|$. Since $p$ lies entirely inside $R(a, b)$ and consists of edges pointing towards $b$, we have that $p$ is an $x y$-monotone path (i.e., any line parallel to a coordinate axis intersects $p$ in at most one point). It follows that $|p| \leq|a c|+|c b|$, which is bounded above by $|a b| \sqrt{2}$.

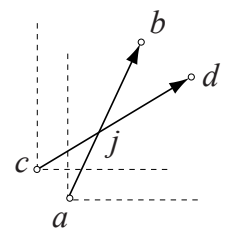

Fig. 4. Lemma 3: if $a b$ and $c d$ cross, they cannot both be in $Y_{4}$.

Lemma 3. Let $a, b, c, d \in V$ be four disjoint nodes such that $\overrightarrow{a b}, \overrightarrow{c d} \in Y_{4}, b \in Q_{i}(a)$ and $d \in Q_{i}(c)$, for some $i \in\{1,2,3,4\}$. Then ab and cd cannot cross.

Proof. We may assume without loss of generality that $i=1$ and $c$ is to the left of $a$. The proof is by contradiction. Assume that $a b$ and $c d$ cross each other. Let $j$ be the intersection point between $a b$ and $c d$ (see Fig. 4). Since $j \in Q_{1}(a) \cap Q_{1}(c)$, it follows that $d \in Q_{1}(a)$ and $b \in Q_{1}(c)$. Thus $|a b| \leq|a d|$, because otherwise, $\overrightarrow{a b}$ cannot be in $Y_{4}$. By Proposition 1 applied to the quadrilateral $a d b c$,

$$
|a d|+|c b|<|a b|+|c d| \text {. }
$$

This along with $|a b| \leq|a d|$ implies that $|c b|<|c d|$, contradicting that $\overrightarrow{c d} \in Y_{4}$.

The next four lemmas (4-7) each concern a pair of crossing $Y_{4}$ edges, culminating (in Lemma 8) in the conclusion that there is a short path in $Y_{4}$ between a pair of endpoints of those edges. We choose to defer the proofs of lemmas 4-6 to the appendix, for a better understanding of the logical flow of our analysis.

Lemma 4. Let $a, b, c$ and $d$ be four disjoint nodes in $V$ such that $\overrightarrow{a b}, \overrightarrow{c d} \in Y_{4}$, and $a b$ crosses $c d$. Then ( $i)$ the ratio between the shortest side and the longer diagonal of the quadrilateral acbd is no greater than $1 / \sqrt{2}$, and (ii) the shortest side of the quadrilateral acbd is strictly shorter than either diagonal.

Lemma 5. Let $a, b, c, d$ be four distinct nodes in $V$, with $c \in Q_{1}(a)$, such that (i) $\overrightarrow{a b} \in Q_{1}(a)$ and $\overrightarrow{c d} \in Q_{2}(c)$ are in $Y_{4}$ and cross each other, and (ii) ad is a shortest 
side of quadrilateral acbd. Then $\mathcal{P}_{R}(a \rightarrow d)$ and $\mathcal{P}_{R}(d \rightarrow a)$ have a nonempty intersection.

Lemma 6. Let $a, b, c, d$ be four distinct nodes in $V$, with $c \in Q_{1}(a)$, such that (i) $\overrightarrow{a b} \in Q_{1}(a)$ and $\overrightarrow{c d} \in Q_{3}(c)$ are in $Y_{4}$ and cross each other, and (ii) ad is a shortest side of quadrilateral acbd. Then $\mathcal{P}_{R}(d \rightarrow a)$ does not cross ab.

The next lemma relies on all of Lemmas 2-6.

Lemma 7. Let $a, b, c, d \in V$ be four distinct nodes such that $\overrightarrow{a b} \in Y_{4}$ crosses $\overrightarrow{c d} \in Y_{4}$, and let $x y$ be a shortest side of the quadrilateral acbd. Then there exist two paths $\mathcal{P}_{x}$ and $\mathcal{P}_{y}$ in $Y_{4}$, where $\mathcal{P}_{x}$ has $x$ as an endpoint and $\mathcal{P}_{y}$ has $y$ as an endpoint, with the following properties:

(i) $\mathcal{P}_{x}$ and $\mathcal{P}_{y}$ have a nonempty intersection.

(ii) $\left|\mathcal{P}_{x}\right|+\left|\mathcal{P}_{y}\right| \leq 3 \sqrt{2}|x y|$.

(iii) Each edge on $\mathcal{P}_{x} \cup \mathcal{P}_{y}$ is no longer than $|x y|$.

Proof. Assume without loss of generality that $b \in Q_{1}(a)$. We discuss the following exhaustive cases:

(1) $c \in Q_{1}(a)$, and $d \in Q_{1}(c)$. In this case, $a b$ and $c d$ cannot cross each other (by Lemma 3), so this case is finished.

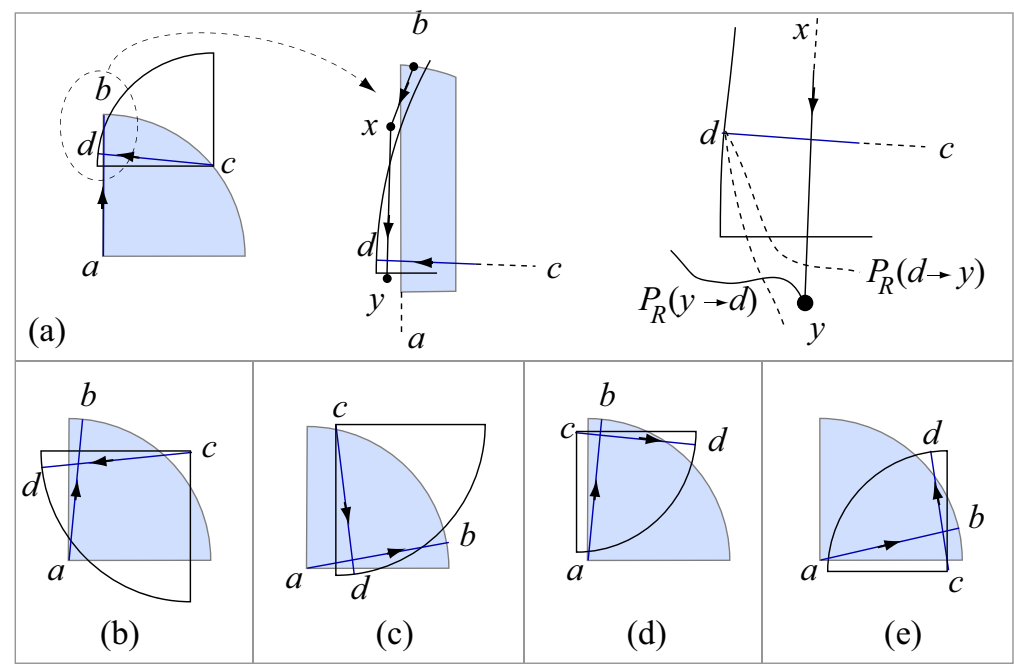

Fig. 5. Lemma 7: (a, b) $c \in Q_{1}(a)(\mathrm{c}) c \in Q_{2}(a)(\mathrm{d}) c \in Q_{4}(a)$.

(2) $c \in Q_{1}(a)$, and $d \in Q_{2}(c)$, as in Fig. 5a. Since $\overrightarrow{a b} \in Y_{4},|a b| \leq|a c|$. Since $a b$ crosses $c d$, and $|a b| \leq|a c|, b \in Q_{2}(c)$. Since $\overrightarrow{c d} \in Y_{4},|c d| \leq|c b|$. These along 
with Lemma 4 imply that $a d$ and $d b$ are the only candidates for a shortest edge of $a c b d$. Assume first that $a d$ is a shortest edge of $a c b d$. By Lemma 3, $\mathcal{P}_{a}=\mathcal{P}_{R}(a \rightarrow d)$ does not cross $c d$, because $\mathcal{P}_{a} \in Q_{2}(a)$ and $c d \in Q_{2}(c)$ are in the quadrants of identical indices. It follows from Lemma 5 that $\mathcal{P}_{a}$ and $\mathcal{P}_{d}=\mathcal{P}_{R}(d \rightarrow a)$ have a nonempty intersection. Furthermore, by Lemma 2 , $\left|\mathcal{P}_{a}\right| \leq|a d| \sqrt{2}$ and $\left|\mathcal{P}_{d}\right| \leq|a d| \sqrt{2}$, and no edge on these paths is longer than $|a d|$, proving the lemma true for this case. Consider now the case when $d b$ is a shortest edge of $a c b d$ (see Fig. 5a). Note that $d$ is below $b$ (otherwise, $d \in Q_{2}(c)$ and $|c d|>|c b|)$ and, therefore, $b \in Q_{1}(d)$. By Lemma $3, \mathcal{P}_{d}=\mathcal{P}_{R}(d \rightarrow b)$ does not cross $a b$, because $\mathcal{P}_{d} \in Q_{1}(d)$ and $a b \in Q_{1}(a)$. If $\mathcal{P}_{b}=\mathcal{P}_{R}(b \rightarrow d)$ does not cross $c d$, then $\mathcal{P}_{b}$ and $\mathcal{P}_{d}$ have a nonempty intersection, proving the lemma true for this case. Otherwise, there exists $\overrightarrow{x y} \in \mathcal{P}_{R}(b \rightarrow d)$ that crosses $c d$ (see Fig. 5a). Define

$$
\begin{aligned}
& \mathcal{P}_{b}=\mathcal{P}_{R}(b \rightarrow d) \oplus \mathcal{P}_{R}(y \rightarrow d) \\
& \mathcal{P}_{d}=\mathcal{P}_{R}(d \rightarrow y) .
\end{aligned}
$$

By Lemma $3, \mathcal{P}_{R}(y \rightarrow d)$ does not cross $c d$, because they are both in quadrant $Q_{2}$. Then $\mathcal{P}_{b}$ and $\mathcal{P}_{d}$ must have a nonempty intersection. We now show that $\mathcal{P}_{b}$ and $\mathcal{P}_{d}$ satisfy conditions (i) and (iii) of the lemma. Proposition 1 applied on the quadrilateral $x d y c$ tells us that $|x c|+|y d|<|x y|+|c d|$. We also have that $|c x| \geq|c d|$, since $\overrightarrow{c d} \in Y_{4}$ and $x$ is in the same quadrant of $c$ as $d$. This along with the inequality above implies $|y d|<|x y|$. Because $x y \in \mathcal{P}_{R}(b \rightarrow d)$, by Lemma 2 we have that $|x y| \leq|b d|$, which along with the previous inequality shows that $|y d|<|b d|$. This along with Lemma 2 shows that condition (iii) of the lemma is satisfied. Furthermore, $\left|\mathcal{P}_{R}(y \rightarrow d)\right| \leq|y d| \sqrt{2}$ and $\left|\mathcal{P}_{R}(d \rightarrow y)\right| \leq|y d| \sqrt{2}$. It follows that $\left|\mathcal{P}_{b}\right|+\left|\mathcal{P}_{d}\right| \leq 3 \sqrt{2}|b d|$.

(3) $c \in Q_{1}(a)$, and $d \in Q_{3}(c)$, as in Fig. 5b. Then $|a c| \geq \max \{a b, c d\}$, and by Lemma $4 a c$ is not a shortest edge of $a c b d$. The case when $b d$ is a shortest edge of $a c b d$ is settled by Lemmas 3 and 2: Lemma 3 tells us that $\mathcal{P}_{d}=\mathcal{P}_{R}(d \rightarrow b)$ does not cross $a b$, (because they are both in $\left.Q_{1},\right)$ and $\mathcal{P}_{b}=\mathcal{P}_{R}(b \rightarrow d)$ does not cross $c d$ (because they are both in $Q_{3}$ ). It follows that $\mathcal{P}_{d}$ and $\mathcal{P}_{b}$ have a nonempty intersection. Furthermore, Lemma 2 guarantees that $\mathcal{P}_{d}$ and $\mathcal{P}_{b}$ satisfy conditions (ii) and (iii) of the lemma. Consider now the case when $a d$ is a shortest edge of $a c b d$; the case when $b c$ is shortest is symmetric. By Lemma 6 , $\mathcal{P}_{R}(d \rightarrow a)$ does not cross $a b$. If $\mathcal{P}_{R}(a \rightarrow d)$ does not cross $c d$, then this case is settled: $\mathcal{P}_{d}=\mathcal{P}_{R}(d \rightarrow a)$ and $\mathcal{P}_{a}=\mathcal{P}_{R}(a \rightarrow d)$ satisfy the three conditions of the lemma. Otherwise, let $\overrightarrow{x y} \in \mathcal{P}_{R}(a \rightarrow d)$ be the edge crossing $c d$. Arguments similar to the ones used in case 1 above show that $\mathcal{P}_{a}=\mathcal{P}_{R}(a \rightarrow d) \oplus \mathcal{P}_{R}(y \rightarrow d)$ and $\mathcal{P}_{d}=\mathcal{P}_{R}(d \rightarrow y)$ are two paths that satisfy the conditions of the lemma.

(4) $c \in Q_{1}(a)$, and $d \in Q_{4}(c)$, as in Fig. 5c. Note that a horizontal reflection of Fig. 5 c, followed by a rotation of $\pi / 2$, depicts a case identical to case (2), Fig. $5 \mathrm{a}$, which has already been settled. 
(5) $c \in Q_{2}(a)$, as in Fig. 5d. Note that Fig. 5d rotated by $\pi / 2$ depicts a case identical to case (2), Fig. 5a (with the roles of $a b$ and $c d$ switched), which has already been settled.

(6) $c \in Q_{3}(a)$. Then it must be that $d \in Q_{1}(c)$, otherwise $c d$ cannot cross $a b$. By Lemma 3 however, $a b$ and $c d$ may not cross, unless one of them is not in $Y_{4}$.

(7) $c \in Q_{4}(a)$. By Lemma 3, $d$ may not lie in $Q_{1}(c)$, therefore $d$ must be in $Q_{2}(c)$, as in Fig. 5e. Note that a vertical reflection of Fig. 5e depicts a case identical to case (2), Fig. 5a (with the roles of $a b$ and $c d$ switched), so this case is settled as well.

We are now ready to establish the main lemma of this section, showing that there is a short path between the endpoints of two intersecting edges in $Y_{4}$.

Lemma 8. Let $a, b, c, d \in V$ be four distinct nodes such that $\overrightarrow{a b} \in Y_{4}$ crosses $\overrightarrow{c d} \in Y_{4}$, and let $x y$ be a shortest side of the quadrilateral acbd. Then $Y_{4}$ contains a path $p(x, y)$ connecting $x$ and $y$, of length $|p(x, y)| \leq \frac{6}{\sqrt{2}-1} \cdot|x y|$. Furthermore, no edge on $p(x, y)$ is longer than $|x y|$.

Proof. Let $\mathcal{P}_{x}$ and $\mathcal{P}_{y}$ be the two paths whose existence in $Y_{4}$ is guaranteed by Lemma 7. By condition (iii) of Lemma 7, no edge on $\mathcal{P}_{x}$ and $\mathcal{P}_{y}$ is longer than $|x y|$. By condition (i) of Lemma $7, \mathcal{P}_{x}$ and $\mathcal{P}_{y}$ have a nonempty intersection. If $\mathcal{P}_{x}$ and $\mathcal{P}_{y}$ share a node $u \in V$, then the path $p(x, y)=\mathcal{P}_{x}[x, u] \oplus \mathcal{P}_{y}[y, u]$ is a path from $x$ to $y$ in $Y_{4}$ no longer than $3 \sqrt{2}|x y|$; the length restriction follows from guarantee (ii) of Lemma 7. Otherwise, let $\overrightarrow{a^{\prime} b^{\prime}} \in \mathcal{P}_{x}$ and $\overrightarrow{c^{\prime} d^{\prime}} \in \mathcal{P}_{y}$ be two edges crossing each other. Let $x^{\prime} y^{\prime}$ be a shortest side of the quadrilateral $a^{\prime} c^{\prime} b^{\prime} d^{\prime}$, with $x^{\prime} \in \mathcal{P}_{x}$ and $y^{\prime} \in \mathcal{P}_{y}$. Lemma 7 tells us that $\left|a^{\prime} b^{\prime}\right| \leq|x y|$ and $\left|c^{\prime} d^{\prime}\right| \leq|x y|$. These along with Lemma 4 imply that

$$
\left|x^{\prime} y^{\prime}\right| \leq|x y| / \sqrt{2}
$$

This enables us to derive a recursive formula for computing a path $p(x, y) \in Y_{4}$ as follows:

$$
p(x, y)= \begin{cases}x, & \text { if } x=y \\ \mathcal{P}_{x}\left[x, x^{\prime}\right] \oplus \mathcal{P}_{y}\left[y, y^{\prime}\right] \oplus p\left(x^{\prime}, y^{\prime}\right), & \text { if } x \neq y\end{cases}
$$

Next we use induction on the length of $x y$ to prove the claim of the lemma. The base case corresponds to $x=y$. In this case $p(x, y)$ degenerates to a point and $|p(x, y)|=0$. To prove the inductive step, pick a shortest side $x y$ of a quadrilateral acbd, with $\overrightarrow{a b}, \overrightarrow{c d} \in Y_{4}$ crossing each other, and assume that the lemma holds for all such sides shorter than $x y$. Let $p(x, y)$ be the path determined recursively as in $(9)$. By the inductive hypothesis, we have that $p\left(x^{\prime}, y^{\prime}\right)$ contains no edges longer than $\left|x^{\prime} y^{\prime}\right| \leq|x y|$, and

$$
\left|p\left(x^{\prime}, y^{\prime}\right)\right| \leq \frac{6}{\sqrt{2}-1}\left|x^{\prime} y^{\prime}\right| \leq \frac{6}{2-\sqrt{2}}|x y|
$$


This latter inequality follows from (8). Also recall that no edge on $\mathcal{P}_{x}$ and $\mathcal{P}_{y}$ is longer than $|x y|$, which together with formula (9) and the arguments above, implies that no edge on $p(x, y)$ is longer than $|x y|$. Substituting inequalities 10 and (ii) from Lemma 7 in formula (9) yields

$$
|p(x, y)| \leq\left(3 \sqrt{2}+\frac{6}{2-\sqrt{2}}\right) \cdot|x y|=\frac{6}{\sqrt{2}-1} \cdot|x y| .
$$

This completes the proof.

\section{4. $Y_{4}^{\infty}$ and $Y_{4}$}

The final step of our analysis is to prove that every individual edge of $Y_{4}^{\infty}$ is spanned by a short path in $Y_{4}$. This, along with the result of Theorem 1, establishes that $Y_{4}$ is a spanner.

Fix an edge $\overrightarrow{a b} \in Y_{4}^{\infty}$. Call an edge or a path $t$-short (with respect to $|a b|$ ) if its length is within a constant factor $t$ of $|a b|$. In our proof that $a b$ is spanned by a $t$-short path in $Y_{4}$, we will make use of the following three statements (proved in the Appendix).

S1 If $x y$ is $t$-short, then $\mathcal{P}_{R}(x \rightarrow y)$, and therefore its reverse, $\mathcal{P}_{R}^{-1}(x \rightarrow y)$ are $t \sqrt{2}$-short by Lemma 2 .

S2 If $x y \in Y_{4}$ is $t_{1}$-short and $z w \in Y_{4}$ is $t_{2}$-short, and if $x y$ intersects $z w$, Lemma 4(ii) and Lemma 8 show that there is a $t_{3}$-short path between any two of the endpoints of these edges, with $t_{3}=t_{1}+t_{2}+3(2+\sqrt{2}) \max \left(t_{1}, t_{2}\right)$.

S3 If $p(x, y)$ is a $t_{1}$-short path and $p(z, w)$ is a $t_{2}$-short path and these two paths intersect, then by $\mathbf{S} \mathbf{2}$ there is a $t_{3}$-short path $P$ between any two of the endpoints of these paths, with $t_{3}=t_{1}+t_{2}+3(2+\sqrt{2}) \max \left(t_{1}, t_{2}\right)$.

Lemma 9. Fix an edge $a b \in Y_{4}^{\infty}$. There is a path $p(a, b) \in Y_{4}$ between $a$ and $b$, of length $|p(a, b)| \leq t|a b|$, for $t=26+23 \sqrt{2}$.

Proof. For the sake of clarity, we only prove here that there is a short path $p(a, b)$ between $a$ and $b$, and and defer the calculation of the actual stretch factor $t$ to the Appendix. We refer to an edge or a path as short if its length is within a constant factor of $|a b|$. Assume without loss of generality that $\overrightarrow{a b} \in Q_{1}(a)$. If $\overrightarrow{a b} \in Y_{4}$, then $p(a, b)=a b$ and the proof is finished. So assume the opposite, and let $\overrightarrow{a c}$ be the edge in $Y_{4}$ that lies in $Q_{1}(a)$; since $Q_{1}(a)$ is nonempty, $\overrightarrow{a c}$ exists. Because $\overrightarrow{a c} \in Y_{4}$ and $b$ is in the same quadrant of $a$ as $c$, we have that

$$
\begin{aligned}
& |a c| \leq|a b| \\
& |b c|<|a c| \sqrt{2}
\end{aligned}
$$

Inequality (ii) above follows immediately from the Law of Cosines, which implies that $|b c|^{2}<|a b|^{2}+|a c|^{2}$ (because the angle formed by $a b$ and $a c$ is strictly less than $\pi / 2$ ), and the fact that $|a c| \leq|a b|$. Thus both $a c$ and $b c$ are short. And this 
in turn implies that $\mathcal{P}_{R}(b \rightarrow c)$ is short by $\mathbf{S 1}$. We next focus on $\mathcal{P}_{R}(b \rightarrow c)$. For simplicity, we assume that $a c$ is counterclockwise of $a b$; the situation when $a c$ lies clockwise of $a b$ is symmetrical. Let $b^{\prime} \notin R(b, c)$ be the other endpoint of $\mathcal{P}_{R}(b \rightarrow c)$. We distinguish three cases.

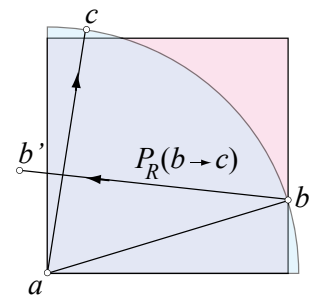

(a)

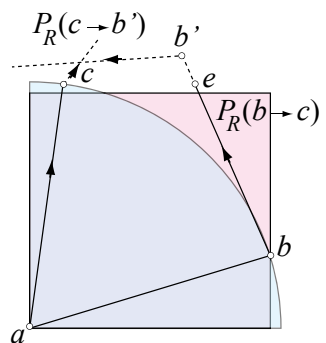

(b)



(c)

Fig. 6. Lemma 9: (a) Case 1: $\mathcal{P}_{R}(b \rightarrow c)$ and $a c$ have a nonempty intersection. (b) Case 2: $\mathcal{P}_{R}\left(b^{\prime} \rightarrow a\right)$ and $a b$ have an empty intersection. (c) Case 3: $\mathcal{P}_{R}\left(b^{\prime} \rightarrow a\right)$ and $a b$ have a non-empty intersection.

Case 1: $\mathcal{P}_{R}(b \rightarrow c)$ and $a c$ intersect (see Fig. 6a). Then by $\mathbf{S 3}$ there is a short path $p(a, b)$ between $a$ and $b$.

Case 2: $\mathcal{P}_{R}(b \rightarrow c)$ and $a c$ do not intersect, and $\mathcal{P}_{R}\left(b^{\prime} \rightarrow a\right)$ and $a b$ do not intersect (see Fig. 6b). Note that because $b^{\prime}$ is the endpoint of the short path $\mathcal{P}_{R}(b \rightarrow c)$, the triangle inequality on $\triangle a b b^{\prime}$ implies that $a b^{\prime}$ is short, and therefore $\mathcal{P}_{R}\left(b^{\prime} \rightarrow a\right)$ is short, by $\mathbf{S 1}$. We consider two cases:

(i) $\mathcal{P}_{R}\left(b^{\prime} \rightarrow a\right)$ intersects $a c$. Then by $\mathbf{S 3}$ there is a short path $p\left(a, b^{\prime}\right)$. So

$$
p(a, b)=p\left(a, b^{\prime}\right) \oplus \mathcal{P}_{R}^{-1}(b \rightarrow c)
$$

is short.

(ii) $\mathcal{P}_{R}\left(b^{\prime} \rightarrow a\right)$ does not intersect $a c$. Then $\mathcal{P}_{R}\left(c \rightarrow b^{\prime}\right)$ must intersect $\mathcal{P}_{R}(b \rightarrow$ $c) \oplus \mathcal{P}_{R}\left(b^{\prime} \rightarrow a\right)$. Next we establish that $b^{\prime} c$ is short. Let $\overrightarrow{e b^{\prime}}$ be the last edge of $\mathcal{P}_{R}(b \rightarrow c)$, and so incident to $b^{\prime}$ (note that $e$ and $b$ may coincide). Because $\mathcal{P}_{R}(b \rightarrow c)$ does not intersect $a c, b^{\prime}$ and $c$ are in the same quadrant for $e$. It follows that $\left|e b^{\prime}\right| \leq|e c|$ and $\angle b^{\prime} e c<\pi / 2$. These observations along with Proposition 2 for $\triangle b^{\prime} e c$ imply that $\left|b^{\prime} c\right|^{2}<\left|b^{\prime} e\right|^{2}+|e c|^{2} \leq 2|e c|^{2}<2|b c|^{2}$ (this latter inequality uses the fact that $\angle b e c>\pi / 2$, which implies that $|e c|<|b c|)$. It follows that

$$
\left|b^{\prime} c\right| \leq|b c| \sqrt{2} \leq 2|a c| \quad \text { (by (11)ii). }
$$

Thus $b^{\prime} c$ is short, and by $\mathbf{S} \mathbf{1}$ we have that $\mathcal{P}_{R}\left(c \rightarrow b^{\prime}\right)$ is short. Since $\mathcal{P}_{R}\left(c \rightarrow b^{\prime}\right)$ intersects the short path $\mathcal{P}_{R}(b \rightarrow c) \oplus \mathcal{P}_{R}\left(b^{\prime} \rightarrow a\right)$, there is by $\mathbf{S} 3$ a short path 
$p(c, b)$, and so

$$
p(a, b)=a c \oplus p(c, b)
$$

is short.

Case 3: $\mathcal{P}_{R}(b \rightarrow c)$ and $a c$ do not intersect, and $\mathcal{P}_{R}\left(b^{\prime} \rightarrow a\right)$ intersects $a b$ (see Fig. 6c). If $\mathcal{P}_{R}\left(b^{\prime} \rightarrow a\right)$ intersects $a b$ at $a$, then $p(a, b)=\mathcal{P}_{R}(b \rightarrow c) \oplus \mathcal{P}_{R}\left(b^{\prime} \rightarrow a\right)$ is short. So assume otherwise, in which case there is an edge $\overrightarrow{d e} \in \mathcal{P}_{R}\left(b^{\prime} \rightarrow a\right)$ that crosses $a b$. Then $d \in Q_{1}(a), e \in Q_{3}(a) \cup Q_{4}(a)$, and $e$ and $a$ are in the same quadrant for $d$. Note however that $e$ cannot lie in $Q_{3}(a)$, since in that case $\angle d a e>\pi / 2$, which would imply $|d e|>|d a|$, which in turn would imply $\overrightarrow{d e} \notin Y_{4}$. So it must be that $e \in Q_{4}(a)$.

Next we show that $\mathcal{P}_{R}(e \rightarrow a)$ does not cross $a b$. Assume the opposite, and let $\overrightarrow{r s} \in \mathcal{P}_{R}(e \rightarrow a)$ cross $a b$. Then $r \in Q_{4}(a), s \in Q_{1}(a) \cup Q_{2}(a)$, and $s$ and $a$ are in the same quadrant for $r$. Arguments similar to the ones above show that $s \notin Q_{2}(a)$, so $s$ must lie in $Q_{1}(a)$. Let $\delta$ be the $L_{\infty}$ distance from $a$ to $b$. Let $x$ be the projection of $r$ on the horizontal line through $a$. Then

$$
|r s| \geq|r x|+\delta \geq|r x|+|x a|>|r a| \quad \text { (by the triangle inequality) }
$$

Because $a$ and $s$ are in the same quadrant for $r$, the inequality above contradicts $\vec{r} \vec{\xi} \in Y_{4}$.

We have established that $\mathcal{P}_{R}(e \rightarrow a)$ does not cross $a b$. Then $\mathcal{P}_{R}(a \rightarrow e)$ must intersect $\mathcal{P}^{\prime}=d e \oplus \mathcal{P}_{R}(e \rightarrow a)$. Note that de is short because it is in the short path $\mathcal{P}_{R}\left(b^{\prime} \rightarrow a\right)$. Thus ae is short (because $|a e|<|a i|+|e i|<|a b|+|e d|$, where $i$ is the intersection point between $a b$ and $d e)$, and so $\mathcal{P}_{R}(a \rightarrow e)$ and $\mathcal{P}_{R}(e \rightarrow a)$ are short, by $\mathbf{S 1}$. Then the short path $\mathcal{P}_{R}(a \rightarrow e)$ intersects either $d e$ or $\mathcal{P}_{R}(e \rightarrow a)$, each of which is short, and by $\mathbf{S 3}$ there is a short path $p(a, e)$. Then

$$
p(a, b)=p(a, e) \oplus \mathcal{P}_{R}^{-1}\left(b^{\prime} \rightarrow a\right) \oplus \mathcal{P}_{R}^{-1}(b \rightarrow c)
$$

is short. Straightforward calculations detailed in the appendix show that, in each of these cases, the stretch factor for $p(a, b)$ does not exceed $26+23 \sqrt{2}$.

Our main result follows immediately from Theorem 1 and Lemma 9:

Theorem 2. $Y_{4}$ is a $t$-spanner, for $t \geq 8 \sqrt{2}(26+23 \sqrt{2})$.

\section{Conclusion}

Our results settle a long-standing open problem, asking whether $Y_{4}$ is a spanner or not. We answer this question positively, and establish a loose stretch factor of $8 \sqrt{2}(26+23 \sqrt{2})$. Finding tighter stretch factors for both $Y_{4}^{\infty}$ and $Y_{4}$ remain interesting open problems. Establishing whether or not $Y_{5}$ is a spanner is also open. 
Acknowledgements. We thank the reviewers for their careful reading and useful comments. The first author was supported by NSERC. The second author was supported in part by NSF grant CCF-0728909 and by Villanova's Center of Excellence in Enterprise Technology.

\section{References}

1. P. Bose, M. Damian, K. Douïeb, J. O'Rourke, B. Seamone, M. Smid and S. Wuhrer, $\pi / 2$-Angle Yao Graphs are Spanners, Technical Report (2010) arXiv:1001.2913v1.

2. P. Bose, A. Maheshwari, G. Narasimhan, M. Smid and N. Zeh, Approximating geometric bottleneck shortest paths, Computational Geometry: Theory and Applications (2004) 29:233-249.

3. M. Damian, N. Molla and V. Pinciu, Spanner properties of $\pi / 2$-angle Yao graphs, in Proc. of the 25th European Workshop on Computational Geometry (March 2009) pp. 21-24.

4. M. Damian and K. Raudonis, Yao graphs span Theta graphs, in Proc. of the 4 th Annual International Conference on Combinatorial Optimization and Applications (December 2010) pp. 181-194.

5. M. Fischer, T. Lukovszki and M. Ziegler, Geometric searching in walkthrough animations with weak spanners in real time, in Proc. of the 6th Annual European Symposium on Algorithms (1998) pp. 163-174.

6. N. Molla, Yao spanners for wireless ad hoc networks, M.S. Thesis, Department of Computer Science, Villanova University (December 2009).

7. J.W. Green, A note on the chords of a convex curve, Portugaliae Mathematica (1951) 10(3):121-123.

8. A.C.-C. Yao, On constructing minimum spanning trees in $k$-dimensional spaces and related problems, SIAM Journal on Computing (1982) 11(4):721-736. 


\section{Appendix}

\subsection{Proof of Lemma 4}

For any node $a \in V$, let $D(a, r)$ denote the open disk centered at $a$ of radius $r$, and let $\partial D(a, r)$ denote the boundary of $D(a, r)$.

Proof. The first part of the lemma is a well-known fact that holds for any quadrilateral (see Ref. ${ }^{7}$, for instance). For the second part of the lemma, let $a b$ be the shorter of the diagonals of $a c b d$, and assume without loss of generality that $\overrightarrow{a b} \in Q_{1}(a)$. Imagine two disks $D_{a}=D(a,|a b|)$ and $D_{b}=D(b,|a b|)$, as in Fig. 7a. If either $c$ or $d$ belongs to $D_{a} \cup D_{b}$, then the lemma follows: a shortest quadrilateral edge is shorter than $|a b|$.

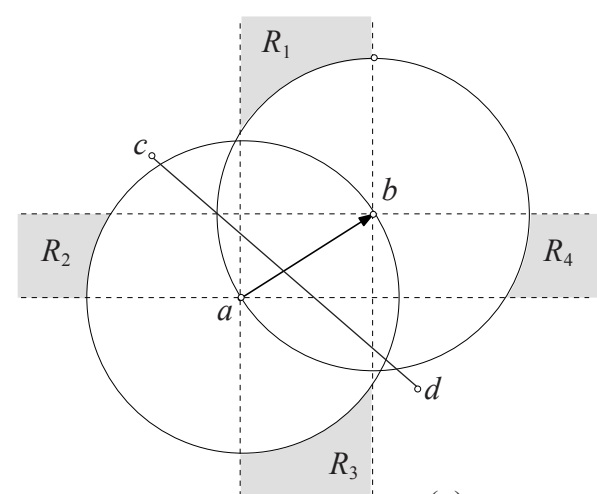

(a)

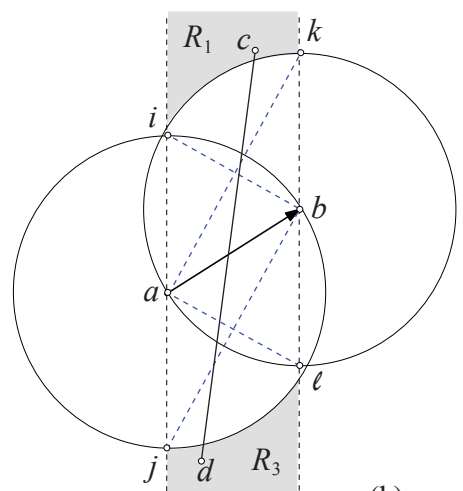

(b)

Fig. 7. Lemma 4 (a) $c \notin R_{1} \cup R_{2} \cup R_{3} \cup R_{4}$ (b) $c \in R_{1}$.

So suppose that neither $c$ nor $d$ lies in $D_{a} \cup D_{b}$. In this case, we use the fact that $c d$ crosses $a b$ to show that $\overrightarrow{c d}$ cannot be an edge in $Y_{4}$. Define the following regions (see Fig. 7a):

$$
\begin{aligned}
& R_{1}=\left(Q_{1}(a) \cap Q_{2}(b)\right) \backslash\left(D_{a} \cup D_{b}\right) \\
& R_{2}=\left(Q_{2}(a) \cap Q_{3}(b)\right) \backslash\left(D_{a} \cup D_{b}\right) \\
& R_{3}=\left(Q_{4}(a) \cap Q_{3}(b)\right) \backslash\left(D_{a} \cup D_{b}\right) \\
& R_{4}=\left(Q_{1}(a) \cap Q_{4}(b)\right) \backslash\left(D_{a} \cup D_{b}\right) .
\end{aligned}
$$

If the node $c$ is not inside any of the regions $R_{i}$, for $i=\{1,2,3,4\}$, then the nodes $a$ and $b$ are in the same quadrant of $c$ as $d$. In this case, note that either $\angle c a d>\pi / 2$ or $\angle c b d>\pi / 2$, which implies that either $|c a|$ or $|c b|$ is strictly smaller than $|c d|$. These together show that $\overrightarrow{c d} \notin Y_{4}$.

So assume that $c$ is in $R_{i}$ for some $i \in\{1,2,3,4\}$. In this situation, the node $d$ must lie in the region $R_{j}$, with $j=(i+2) \bmod 4$ (with the understanding that 
$R_{0}=R_{4}$ ), because otherwise, either (i) $a$ and $d$ are in the same quadrant of $c$ and $|c a|<|c d|$ or (ii) $b$ and $d$ are in the same quadrant of $c$ and $|c b|<|c d|$. Either case contradicts the fact $\overrightarrow{c d} \in Y_{4}$. Consider now the case $c \in R_{1}$ and $d \in R_{3}$; the other cases are treated similarly. Let $i$ and $j$ be the intersection points between $D_{a}$ and the vertical line through $a$. Similarly, let $k$ and $\ell$ be the intersection points between $D_{b}$ and the vertical line through $b$ (see Fig. 7b). Since $i j$ is a diameter of $D_{a}$, we have that $\angle i b j=\pi / 2$ and similarly $\angle k a l=\pi / 2$. Also note that $\angle c b d \geq \angle i b j=\pi / 2$, meaning that $|c d|>|c b|$. Similarly, $\angle c a d \geq \angle k a l=\pi / 2$, meaning that $|c d|>|c a|$. These along with the fact that at least one of $a$ and $b$ is in the same quadrant for $c$ as $d$, imply that $\overrightarrow{c d} \notin Y_{4}$. This completes the proof.

\subsection{Proof of Lemma 5}

Proof. The proof consists of two parts showing that the following claims hold: (I) $d \in Q_{2}(a)$ and $(\mathrm{II}) \mathcal{P}_{R}(d \rightarrow a)$ does not cross $a b$. Before we prove these two claims, let us argue that they are sufficient to prove the lemma. Lemma 3 and claim (I) imply that $\mathcal{P}_{R}(a \rightarrow d)$ cannot cross $c d$, because $\mathcal{P}_{R}(a \rightarrow d) \in Q_{2}(a)$ and $c d \in Q_{2}(c)$ are in quadrants of identical indices. As a result, $\mathcal{P}_{R}(a \rightarrow d)$ intersects the left side of the rectangle $R(d, a)$. Consider the last edge $\overrightarrow{x y}$ of the path $\mathcal{P}_{R}(d \rightarrow a)$. If this edge crosses the right side of $R(a, d)$, then claim (II) implies that $y$ is in the wedge bounded by $a b$ and the upwards vertical ray starting at $a$; this further implies that $|a y|<|a b|$, contradicting the fact that $\overrightarrow{a b}$ is an edge in $Y_{4}$. Therefore, $\overrightarrow{x y}$ intersects the bottom side of $R(d, a)$, and the lemma follows (see Fig. 8b).

To prove the first claim (I), we observe that the lemma assumptions imply that $d \in Q_{1}(a) \cup Q_{2}(a)$. Therefore, it suffices to prove that $d$ is not in $Q_{1}(a)$. Assume to the contrary that $d \in Q_{1}(a)$. Since $c \in Q_{1}(a)$, it must be that $b \in Q_{2}(c)$; otherwise, $\angle a c b \geq \pi / 2$, which implies $|a b|>|a c|$, contradicting the fact that $\overrightarrow{a b} \in Y_{4}$. Let $i$ and $j$ be the intersection points between $c d$ and $\partial D(a,|a b|)$, where $i$ is to the left of $j$. Since $\angle d b c \geq \angle i b j>\pi / 2$, we have $|c b|<|c d|$. This, together with the fact that $b$ and $d$ are in the same quadrant $Q_{2}(c)$, contradicts the assumption that $\overrightarrow{c d}$ is an edge in $Y_{4}$. This completes the proof of claim (I).

Next we prove claim (II) by contradiction. Thus, we assume that there is an edge $\overrightarrow{x y}$ on the path $\mathcal{P}_{R}(d \rightarrow a)$ that crosses $a b$. Then necessarily $x \in R(a, d)$ and $y \in Q_{1}(a) \cup Q_{4}(a)$. If $y \in Q_{4}(a)$, then $\angle x a y>\pi / 2$, meaning that $|x y|>|x a|$, a contradiction to the fact that $\overrightarrow{x y} \in Y_{4}$. Thus, it must be that $y \in Q_{1}(a)$, as in Fig. 8a. This implies that $|a b| \leq|a y|$, because $\overrightarrow{a b} \in Y_{4}$.

The contradiction to our assumption that $\overrightarrow{x y}$ crosses $a b$ will be obtained by proving that $|x y|>|x a|$. Indeed, this inequality contradicts the fact that $\overrightarrow{x y} \in Y_{4}$, because both $a$ and $y$ are in $Q_{4}(x)$, and $Y_{4}$ would have picked $\overrightarrow{x a}$ in place of $\overrightarrow{x y}$.

Let $\delta$ be the distance from $x$ to the horizontal line through $a$. Our intermediate goal is to show that

$$
\delta \leq|a b| / \sqrt{2} .
$$




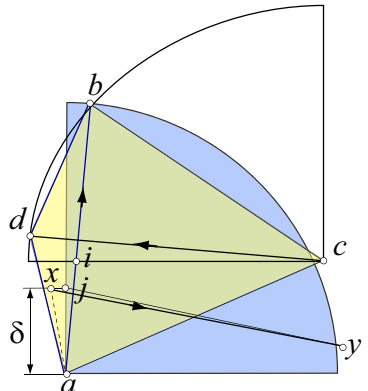

(a)

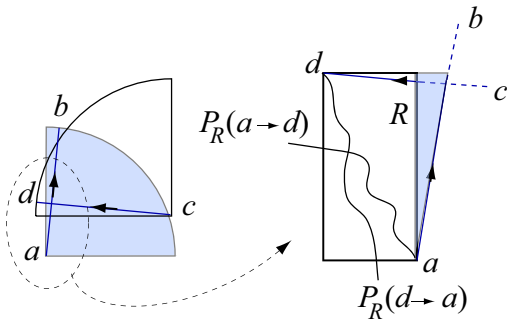

(b)

Fig. 8. (a) Lemma 5: $x y \in \mathcal{P}_{R}(d \rightarrow a)$ cannot cross $a b$.

We claim that $\angle a c b<\pi / 2$. Indeed, if this is not the case, then $|a c|<|a b|$, contradicting the fact that $\overrightarrow{a b}$ is an edge in $Y_{4}$. By a similar argument, and using the fact that $\overrightarrow{c d}$ is an edge in $Y_{4}$, we obtain the inequality $\angle c b d<\pi / 2$. We now consider two cases, depending on the relative lengths of $a c$ and $c b$.

(1) Assume first that $|a c|>|c b|$. If $\angle c a d \geq \pi / 2$, then $|c d| \geq|a c|>|c b|$, contradicting the fact that $\overrightarrow{c d}$ is an edge in $Y_{4}$ (recall that $b$ and $d$ are in the same quadrant of $c$ ). Therefore, we have $\angle c a d<\pi / 2$. So far we have established that three angles of the convex quadrilateral $a c b d$ are acute. It follows that the fourth one $(\angle a d b)$ is obtuse. Proposition 2 applied to $\triangle a d b$ tells us that

$$
|a b|^{2}>|a d|^{2}+|d b|^{2} \geq 2|a d|^{2},
$$

where the latter inequality follows from the assumption that $a d$ is a shortest side of $a c b d$ (and, therefore, $|d b| \geq|a d|$ ). Thus, we have that $|a d| \leq|a b| / \sqrt{2}$. This along with the fact that $x \in R(a, d)$ implies inequality (13).

(2) Assume now that $|a c| \leq|c b|$. Let $i$ be the intersection point between $a b$ and the horizontal line through $c$ (refer to Fig. 8a). Note that $\angle a i c \geq \pi / 2$ and $\angle b i c \leq \pi / 2$ (these two angles sum to $\pi$ ). This along with Proposition 2 applied to triangle $\triangle$ aic shows that

$$
|a c|^{2} \geq|a i|^{2}+|i c|^{2} .
$$

Similarly, Proposition 2 applied to triangle $\triangle$ bic shows that

$$
|b c|^{2} \leq|b i|^{2}+|i c|^{2} .
$$

The two inequalities above along with our assumption that $|a c| \leq|c b|$ imply that $|a i| \leq|b i|$, which in turn implies that $|a i| \leq|a b| / 2$, because $|a i|+|i b|=|a b|$. Since $x$ is below $i$ (otherwise, $|c x|<|c d|$, contradicting the fact that $\overrightarrow{c d}$ is an edge in $Y_{4}$ ), we have $\delta \leq|a i|$. It follows that $\delta \leq|a b| / 2$.

Finally we derive a contradiction using the now established inequality (13). Let $j$ be the orthogonal projection of $x$ onto the vertical line through $a$ (thus $|a j|=\delta$ ). 
Note that $\angle a j y<\pi / 2$, because $y \in Q_{4}(x)$. By Proposition 2 applied to $\triangle a j y$, we have

$$
|a y|^{2}<|a j|^{2}+|j y|^{2}=\delta^{2}+|j y|^{2} .
$$

Since $y$ and $b$ are in the same quadrant of $a$, and since $\overrightarrow{a b} \in Y_{4}$, we have that $|a b| \leq$ $|a y|$. This along with the inequality above and (13) implies that $|j y| \geq|a b| / \sqrt{2} \geq \delta$. By Proposition 2 applied to $\triangle x j y$, we have $|x y|^{2}>|x j|^{2}+|j y|^{2} \geq|x j|^{2}+\delta^{2}=$ $|x j|^{2}+|j a|^{2}=|x a|^{2}$. It follows that $|x y|>|x a|$, contradicting our assumption that $\overrightarrow{x y} \in Y_{4}$.

\subsection{Proof of Lemma 6}

Proof. We first show that $d \notin Q_{3}(a)$. Assume the opposite. Since $c \in Q_{1}(a)$ and
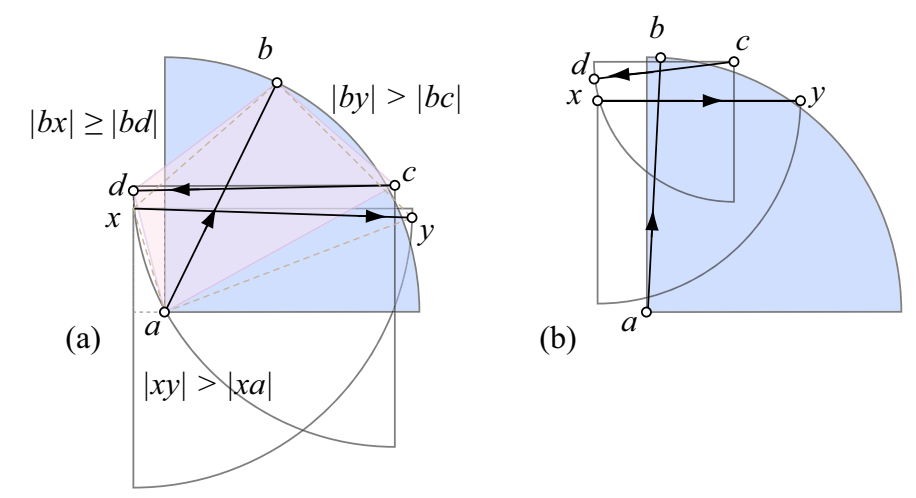

Fig. 9. Lemma 6: (a) $\mathcal{P}_{R}(d \rightarrow a)$ does not cross $a b$. (b) If $a d$ is not the shortest side of $a c b d$, the lemma conclusion might not hold.

$d \in Q_{3}(a)$, we have that $\angle c a d>\pi / 2$. This implies that $|c a|<|c d|$, which along with the fact that $a, d \in Q_{3}(c)$ contradict the fact that $\overrightarrow{c d} \in Y_{4}$. Also note that $d \notin Q_{1}(a)$, since in that case $a b$ and $c d$ could not intersect. In the following we discuss the case $d \in Q_{2}(a)$; the case $d \in Q_{4}(a)$ is symmetric.

A first observation is that $c$ must lie below $b$; otherwise $|c b|\langle|c d|$ (since $\angle c b d\rangle$ $\pi / 2$ ), which would contradict the fact that $\overrightarrow{c d} \in Y_{4}$. We now prove by contradiction that there is no edge in $\mathcal{P}_{R}(d \rightarrow a)$ crossing $a b$. Assume the contrary, and let $\overrightarrow{x y} \in \mathcal{P}_{R}(d \rightarrow a)$ be such an edge. Then necessarily $x \in R(a, d)$ and $\overrightarrow{x y} \in Q_{4}(x)$. Note that $y$ cannot lie below $a$; otherwise $|x a|<|x y|$ (since $\angle x a y>\pi / 2$ ), which would contradict the fact that $\overrightarrow{x y} \in Y_{4}$. Also $y$ must lie outside $D(c,|c d|) \cap Q(c, d)$, otherwise $\overrightarrow{c d}$ could not be in $Y_{4}$. These together show that $y$ sits to the right of $c$. See Fig. 9a. Then the following inequalities regarding the quadrilateral $x a y b$ must hold: 
(i) $|b y|>|b c|$, due to the fact that $\angle b c y>\pi / 2$.

(ii) $|b x| \geq|b d|(|b x|=|b d|$ if $x$ and $d$ coincide). If $x$ and $d$ are distinct, the inequality $|b x|>|b d|$ follows from the fact that $|c x| \geq|c d|$ (since $x$ is outside $D(c,|c d|)$ ), and Proposition 1 applied to the quadrilateral $x c b d$ :

$$
|b d|+|c x|<|b x|+|c d|
$$

Inequalities (i) and (ii) show that $b y$ and $b x$ are longer than sides of the quadrilateral $a c b d$, and so they must be longer than the shortest side of $a c b d$, which by assumption (ii) of the lemma is $a d: \min \{|b x|,|b y|\} \geq|a d| \geq|a x|$ (this latter inequality follows from the fact that $x \in R(d, a))$. Also note that $|a b| \leq|a y|$, since $\overrightarrow{a b} \in Y_{4}$ and $y$ lies in the same quadrant of $a$ as $b$. The fact that both diagonals of $x a y b$ are in $Y_{4}$ enables us to apply Lemma 4(ii) to conclude that ay is not a shortest side of the quadrilateral $x a y b$. Thus $x a$ is a shortest side of the quadrilateral $x a y b$, and we can use Lemma 4(ii) to claim that

$$
|x a|<\min \{|x y|,|a b|\} \leq|x y| .
$$

This contradicts our assumption that $\overrightarrow{x y} \in Y_{4}$.

Fig. 9(b) shows that the claim of the lemma might be false without assumption (ii).

\subsection{Calculations for the stretch factor of $p(a, b)$ in Lemma 9}

We start by computing the stretch factor of the short paths claimed by statements S2 and S3.

S2 If $x y \in Y_{4}$ and $z w \in Y_{4}$ are short, and if $x y$ intersects $z w$, then there is a short path $P$ between any two of the endpoints of these edges, of length

$$
|P| \leq|x y|+|z w|+3(2+\sqrt{2}) \max \{|x y|,|z w|\} .
$$

This upper bound can be derived as follows. Let $i j$ be a shortest side of the quadrilateral $x z y w$. By Lemma $8, Y_{4}$ contains a path $p(i, j)$ no longer than $6(\sqrt{2}+1)|i j|$. By Lemma $4,|i j| \leq \max \{|x y|,|z w|\} / \sqrt{2}$. These together with the fact that $|P| \leq|x y|+|z w|+|p(i, j)|$ yield inequality (14).

S3 Here we prove a tighter version of this statement: If $p(x, y)$ and $p(z, w)$ are short paths that intersect, then there is a short path $P$ between any two of the endpoints of these paths, of length

$$
|P| \leq|p(x, y)|+|p(z, w)|+3(2+\sqrt{2}) \max \{|x y|,|z w|\} .
$$

This follows immediately from $\mathbf{S 2}$ and the fact that no edge of $p(x, y) \cup p(z, w)$ is longer than $\max \{|x y|,|z w|\}$ (by Lemma 8).

Case 1: $\mathcal{P}_{R}(b \rightarrow c)$ and $a c$ intersect. Then by $\mathbf{S 3}$ we have 


$$
\begin{array}{rlrl}
|p(a, b)| & \leq\left|\mathcal{P}_{R}(b, c)\right|+|a c|+3(2+\sqrt{2}) \max \{|b c|,|a c|\} & \\
& \leq \sqrt{2}|b c|+|a c|+3(2+\sqrt{2}) \sqrt{2}|a c| & & \text { (by (7), (11)ii) } \\
& =3(3+2 \sqrt{2})|a c| \leq 3(3+2 \sqrt{2})|a b| & & \text { (by (11)i). }
\end{array}
$$

Case 2(i): $\mathcal{P}_{R}(b \rightarrow c)$ and $a c$ do not intersect; $\mathcal{P}_{R}\left(b^{\prime} \rightarrow a\right)$ and $a b$ do not intersect; and $\mathcal{P}_{R}\left(b^{\prime} \rightarrow a\right)$ intersects $a c$. By S3, there is a short path $p\left(a, b^{\prime}\right)$ of length

$$
\begin{aligned}
\left|p\left(a, b^{\prime}\right)\right| & \leq\left|\mathcal{P}_{R}\left(b^{\prime}, a\right)\right|+|a c|+3(2+\sqrt{2}) \max \left\{\left|b^{\prime} a\right|,|a c|\right\} \\
& \leq\left|b^{\prime} a\right| \sqrt{2}+|a c|+3(2+\sqrt{2}) \max \left\{\left|b^{\prime} a\right|,|a c|\right\} \quad \text { (by (7)). }
\end{aligned}
$$

Next we establish an upper bound on $\left|b^{\prime} a\right|$. By the triangle inequality,

$$
\left|a b^{\prime}\right|<|a c|+\left|c b^{\prime}\right| \leq 3|a c| \quad \text { (by (12)). }
$$

Substituting this inequality in (16) yields

$$
\left|p\left(a, b^{\prime}\right)\right| \leq(19+12 \sqrt{2})|a c| .
$$

Thus $p(a, b)=p\left(a, b^{\prime}\right) \oplus \mathcal{P}_{R}^{-1}(b \rightarrow c)$ is a path in $Y_{4}$ of length

$$
\begin{aligned}
|p(a, b)| & \leq\left|p\left(a, b^{\prime}\right)\right|+|b c| \sqrt{2} & & \text { (by (7)) } \\
& \leq\left|p\left(a, b^{\prime}\right)\right|+2|a c| & & \text { (by (11)ii) } \\
& \leq(21+12 \sqrt{2})|a c| & & \text { (by (18)) } \\
& \leq(21+12 \sqrt{2})|a b| & & \text { (by (11)i). }
\end{aligned}
$$

Case 2(ii): $\mathcal{P}_{R}(b \rightarrow c)$ and $a c$ do not intersect; $\mathcal{P}_{R}\left(b^{\prime} \rightarrow a\right)$ and $a b$ do not intersect; and $\mathcal{P}_{R}\left(b^{\prime} \rightarrow a\right)$ does not intersect $a c$. Then $\mathcal{P}_{R}\left(c \rightarrow b^{\prime}\right)$ must intersect $\mathcal{P}_{R}(b \rightarrow$ $c) \oplus \mathcal{P}_{R}\left(b^{\prime} \rightarrow a\right)$. By $\mathbf{S 3}$ there is a short path $p(c, b)$ of length

$$
\begin{aligned}
|p(c, b)| & \leq\left|\mathcal{P}_{R}\left(c \rightarrow b^{\prime}\right)\right|+\left|\mathcal{P}_{R}(b \rightarrow c)\right|+\left|\mathcal{P}_{R}\left(b^{\prime} \rightarrow a\right)\right|+3(2+\sqrt{2}) \max \left\{\left|c b^{\prime}\right|,|b c|,\left|b^{\prime} a\right|\right\} \\
& \left.\leq\left(\left|c b^{\prime}\right|+|b c|+\left|b^{\prime} a\right|\right) \sqrt{2}+3(2+\sqrt{2}) \max \left\{\left|c b^{\prime}\right|,|b c|,\left|b^{\prime} a\right|\right\} \quad \text { (by }(7)\right) .
\end{aligned}
$$

Inequalities (11)ii, (12) and (17) imply that $\max \left\{\left|c b^{\prime}\right|,|b c|,\left|b^{\prime} a\right|\right\} \leq 3 a c$. Substituting in the above, we get

$$
\begin{aligned}
|p(c, b)| & \leq(2+\sqrt{2}+3) \sqrt{2}|a c|+9(2+\sqrt{2})|a c| \\
& \leq(20+14 \sqrt{2})|a c| \quad \text { (by (11)i). }
\end{aligned}
$$

Thus $p(a, b)=a c \oplus p(c, b)$ is a path in $Y_{4}$ from $a$ to $b$ of length

$$
|p(a, b)| \leq(21+14 \sqrt{2})|a c| \leq(21+14 \sqrt{2})|a b| \quad \text { (by (11)i). }
$$


Case 3: $\mathcal{P}_{R}(b \rightarrow c)$ and $a c$ do not intersect, and $\mathcal{P}_{R}\left(b^{\prime} \rightarrow a\right)$ intersects $a b$. If $\mathcal{P}_{R}\left(b^{\prime} \rightarrow a\right)$ intersects $a b$ at $a$, then $p(a, b)=\mathcal{P}_{R}(b \rightarrow c) \oplus \mathcal{P}_{R}\left(b^{\prime} \rightarrow a\right)$ is clearly short and does not exceed the spanning ratio of the lemma. Otherwise, there is an edge $\overrightarrow{d e} \in \mathcal{P}_{R}\left(b^{\prime} \rightarrow a\right)$ that crosses $a b$, and $\mathcal{P}_{R}(a \rightarrow e)$ intersects $d e \oplus \mathcal{P}_{R}(e \rightarrow a)$ (as established in the proof of Lemma 9). If $\mathcal{P}_{R}(a \rightarrow e)$ intersects de, then by $\mathbf{S} 3$ there is a short path $p(a, e)$ of length

$$
|p(a, e)| \leq\left|\mathcal{P}_{R}(a \rightarrow e)\right|+|d e|+3(2+\sqrt{2}) \max \{|a e|,|d e|\}
$$

Otherwise, if $\mathcal{P}_{R}(a \rightarrow e)$ intersects $\mathcal{P}_{R}(e \rightarrow a)$, then by $\mathbf{S} \mathbf{3}$ there is a short path $p(a, e)$ of length

$$
|p(a, e)| \leq\left|\mathcal{P}_{R}(a \rightarrow e)\right|+\left|\mathcal{P}_{R}(e \rightarrow a)\right|+3(2+\sqrt{2})|a e|
$$

A loose upper bound on $|a e|$ can be obtained by employing Proposition 1 to the quadrilateral aebd: $|a e|+|b d|<|a b|+|d e|<|a b|+\left|a b^{\prime}\right|$. Substituting the upper bound for $a b^{\prime}$ from (17) yields

$$
|a e|<|a b|+3|a c| \leq 4|a b| .
$$

By Lemma 2, $|d e| \leq\left|a b^{\prime}\right|$ (since $d e \in \mathcal{P}_{R}\left(b^{\prime} \rightarrow a\right)$ ), which along with (17) implies

$$
|d e| \leq 3|a b| \text {. }
$$

Substituting inequalities (7), (21) and (22) in (19) yields

$$
|p(a, e)| \leq(27+16 \sqrt{2})|a b| .
$$

Substituting inequalities (7) and (21) in (20) gives

$$
|p(a, e)| \leq(24+20 \sqrt{2})|a b|,
$$

which is a looser upper bound that applies to both cases. Then

$$
p(a, b)=p(a, e) \oplus \mathcal{P}_{R}^{-1}\left(b^{\prime} \rightarrow a\right) \oplus \mathcal{P}_{R}^{-1}(b \rightarrow c)
$$

is a path from $a$ to $b$ of length

$$
\begin{aligned}
|p(a, b)| & \leq(24+20 \sqrt{2})|a b|+3 \sqrt{2}|a b|+2|a b| \quad \text { (by (23), (17), (11)) } \\
& =(26+23 \sqrt{2})|a b| .
\end{aligned}
$$

